\title{
Optimal Feedback Control for Dynamic Systems with State Constraints: An Exact Penalty Approach
}

\author{
Qun Lin • Ryan Loxton • Kok Lay Teo • \\ Yong Hong Wu
}

Received: date / Accepted: date

\begin{abstract}
In this paper, we consider a class of nonlinear dynamic systems with terminal state and continuous inequality constraints. Our aim is to design an optimal feedback controller that minimizes total system cost and ensures satisfaction of all constraints. We first formulate this problem as a semi-infinite optimization problem. We then show that by using a new exact penalty approach, this semi-infinite optimization problem can be converted into a sequence of nonlinear programming problems, each of which can be solved using standard gradient-based optimization methods. We conclude the paper by discussing applications of our work to glider control.
\end{abstract}

Keywords Optimal Control · Semi-Infinite Optimization · State Feedback · Nonlinear Constraints · Exact Penalty Function

Mathematics Subject Classification (2000) 49M37 - 93C10 - 90C30 . $65 \mathrm{~K} 05$

Q. Lin

Department of Mathematics and Statistics, Curtin University, Perth, Western Australia E-mail: q.lin@curtin.edu.au

R. Loxton

Department of Mathematics and Statistics, Curtin University, Perth, Western Australia E-mail: r.loxton@curtin.edu.au

K. L. Teo

Department of Mathematics and Statistics, Curtin University, Perth, Western Australia E-mail: k.l.teo@curtin.edu.au

Y. H. Wu

Department of Mathematics and Statistics, Curtin University, Perth, Western Australia E-mail: y.wu@curtin.edu.au 


\section{Introduction}

Optimal control problems with state constraints arise in a wide range of practical applications, including robotics [6], switching DC-DC power converters [16], zinc sulphate purification [24], and semibatch chemical reactors [28]. State constraints can be classified into two types: terminal state constraints, which depend solely on the final state reached by the system, and continuous inequality constraints, which restrict the state at every point in the time horizon. Of these two types, continuous inequality constraints are by far the most difficult, as they are imposed continuously across the entire time horizon, not just at a single point.

In recent years, several new computational methods for solving optimal control problems with continuous inequality constraints have been proposed. These include the non-smooth Newton's method [6], the constraint transcription method [24], the exact penalty method [26], the lossless convexification method [1], and methods based on interval analysis [28]. Although these methods have proven to be very effective at solving practical problems, they are only capable of producing an open loop control. Such open loop controls may not be robust in practice. Thus, an optimal feedback control, expressed as a function of the current system state, is usually preferred.

The traditional approach for determining an optimal feedback control involves solving the well-known HJB partial differential equation - a difficult task even for unconstrained problems. Thus, in general, computing an optimal feedback control for constrained systems is very challenging. Nevertheless, some partial attempts have been made in the literature. In [10], for example, an optimal PID controller is derived by solving an optimization problem in which the feedback gains are decision variables. This problem contains continuous inequality constraints that arise because of engineering specifications on the controller, such as bounds on the overshoot and rise time. The optimal feedback gains are determined using the constraint transcription technique [17], which is a well-known computational technique for handling state constraints.

Other relevant approaches for solving the feedback control problem include the sensitivity penalization approach for computing robust suboptimal controllers [15,21], and the neighbouring extremal approach [5,7], which invokes rules for updating the optimal control when new information about the system becomes available. Reference [29] describes a feedback control method specifically designed for lunar landing modules. This method is based on a novel combination of the time-scaling and control parameterization techniques [14], the famous Pontryagin's minimum principle [20], and cubic spline approximation [3].

In this paper, we consider a general optimal control problem in which a feedback controller of given structure is optimized by varying certain adjustable parameters. Unlike in $[10,29]$, we do not restrict ourselves to any particular control structure or any particular system - our problem is completely general. We first formulate the optimal feedback control problem as a semiinfinite optimization problem in which the adjustable control parameters are 
decision variables. We then show how to solve this semi-infinite optimization problem using a recently-developed exact penalty method, which has proven to be very effective at handling semi-infinite and discrete constraints $[26,27]$. This exact penalty approach has two key advantages over the constraint transcription method used in [10]: (i) it involves one approximation parameter instead of two; and (ii) it is guaranteed to converge to a local optimal solution (in contrast, the constraint transcription method is only guaranteed to converge if the global solution is obtained at each step, which is unlikely in practice). We demonstrate the effectiveness of our new exact penalty approach by applying it to the optimal control of a hang glider.

\section{Problem Statement}

Consider the following nonlinear control system:

$$
\begin{aligned}
\dot{\boldsymbol{x}}(t) & =\boldsymbol{f}(\boldsymbol{x}(t), \boldsymbol{u}(t)), \quad t \in[0, T], \\
\boldsymbol{x}(0) & =\boldsymbol{x}^{0}
\end{aligned}
$$

where $\boldsymbol{x}(t) \in \mathbb{R}^{n}$ is the state, $\boldsymbol{u}(t) \in \mathbb{R}^{r}$ is the control, $T$ is a given terminal time, $\boldsymbol{x}^{0} \in \mathbb{R}^{n}$ is a given initial state, and $\boldsymbol{f}: \mathbb{R}^{n} \times \mathbb{R}^{r} \rightarrow \mathbb{R}^{n}$ is a given continuously differentiable function.

System (1)-(2) is subject to the following terminal state constraints:

$$
\Psi_{i}(\boldsymbol{x}(T))=0, \quad i=1, \ldots, p,
$$

where $\Psi_{i}: \mathbb{R}^{n} \rightarrow \mathbb{R}, i=1, \ldots, p$ are given continuously differentiable functions.

System (1)-(2) is also subject to the following continuous inequality constraints:

$$
h_{j}(\boldsymbol{x}(t), \boldsymbol{u}(t)) \leq 0, \quad t \in[0, T], \quad j=1, \ldots, q,
$$

where $h_{j}: \mathbb{R}^{n} \times \mathbb{R}^{r} \rightarrow \mathbb{R}, j=1, \ldots, q$ are given continuously differentiable functions. Note that control bounds can be easily incorporated into (4).

Our task is to design an optimal feedback controller for system (1)-(2). We assume that the control takes the following form:

$$
\boldsymbol{u}(t)=\boldsymbol{\varphi}(\boldsymbol{x}(t), \boldsymbol{\zeta}), \quad t \in[0, T]
$$

where $\boldsymbol{\zeta}=\left[\zeta_{1}, \ldots, \zeta_{m}\right]^{\top} \in \mathbb{R}^{m}$ is a vector of feedback control parameters and $\varphi: \mathbb{R}^{n} \times \mathbb{R}^{m} \rightarrow \mathbb{R}^{r}$ is a given continuously differentiable function. The function $\varphi$ governs the structure of the feedback controller (assumed to be given), while the control parameters $\zeta_{k}, k=1, \ldots, m$ are decision variables to be chosen optimally. One of the most common feedback control structures is the linear state feedback control law given by $\boldsymbol{u}(t)=K \boldsymbol{x}(t)$, where $K \in \mathbb{R}^{r \times n}$ is a feedback gain matrix whose elements need to be determined.

The following bound constraints are imposed on the feedback control parameters:

$$
a_{k} \leq \zeta_{k} \leq b_{k}, \quad k=1, \ldots, m,
$$


where $a_{k}$ and $b_{k}, k=1, \ldots, m$ are given constants. Let $\Gamma$ denote the set of all $\boldsymbol{\zeta}=\left[\zeta_{1}, \ldots, \zeta_{m}\right]^{\top} \in \mathbb{R}^{m}$ satisfying $(6)$.

Substituting (5) into (1) gives

$$
\dot{\boldsymbol{x}}(t)=\tilde{\boldsymbol{f}}(\boldsymbol{x}(t), \boldsymbol{\zeta}), \quad t \in[0, T],
$$

where

$$
\tilde{\boldsymbol{f}}(\boldsymbol{x}(t), \boldsymbol{\zeta})=\boldsymbol{f}(\boldsymbol{x}(t), \boldsymbol{\varphi}(\boldsymbol{x}(t), \boldsymbol{\zeta})) .
$$

Consider the system of differential equations (7) with the initial condition (2). Let $\boldsymbol{x}(\cdot \mid \boldsymbol{\zeta})$ denote the solution of this system. Then the terminal constraints (3) become

$$
\Psi_{i}(\boldsymbol{x}(T \mid \boldsymbol{\zeta}))=0, \quad i=1, \ldots, p
$$

Furthermore, the path constraints (4) become

$$
\tilde{h}_{j}(\boldsymbol{x}(t \mid \boldsymbol{\zeta}), \boldsymbol{\zeta}) \leq 0, \quad t \in[0, T], \quad j=1, \ldots, q,
$$

where

$$
\tilde{h}_{j}(\boldsymbol{x}(t \mid \boldsymbol{\zeta}), \boldsymbol{\zeta})=h_{j}(\boldsymbol{x}(t \mid \boldsymbol{\zeta}), \boldsymbol{\varphi}(\boldsymbol{x}(t \mid \boldsymbol{\zeta}), \boldsymbol{\zeta})) .
$$

Let $\Lambda$ denote the set of all $\boldsymbol{\zeta} \in \Gamma$ satisfying (8) and (9).

We now consider the problem of choosing the feedback control parameters $\zeta_{k}, k=1, \ldots, m$ to minimize the total system cost subject to constraints (8) and (9).

Problem P. Choose $\boldsymbol{\zeta} \in \Lambda$ to minimize the cost function

$$
J(\boldsymbol{\zeta})=\Phi(\boldsymbol{x}(T \mid \boldsymbol{\zeta}), \boldsymbol{\zeta})+\int_{0}^{T} \mathcal{L}(\boldsymbol{x}(t \mid \boldsymbol{\zeta}), \boldsymbol{\zeta}) d t
$$

where $\Phi: \mathbb{R}^{n} \times \mathbb{R}^{m} \rightarrow \mathbb{R}$ and $\mathcal{L}: \mathbb{R}^{n} \times \mathbb{R}^{m} \rightarrow \mathbb{R}$ are given continuously differentiable functions.

Problem $\mathrm{P}$ is a nonlinear optimization problem in which a finite number of decision variables (the feedback control parameters) need to be optimized subject to a set of constraints. This is a very difficult optimization problem to solve because each continuous inequality constraint in (9) actually constitutes an infinite number of constraints - one for each point in $[0, T]$. Hence, Problem P can be viewed as a semi-infinite optimization problem. In the next section, we will use a novel exact penalty approach to approximate Problem P by a nonlinear programming problem. 


\section{An Exact Penalty Approach}

Define a constraint violation function on $\Gamma$ as follows:

$$
\Delta(\boldsymbol{\zeta})=\sum_{i=1}^{p} \Psi_{i}(\boldsymbol{x}(T \mid \boldsymbol{\zeta}))^{2}+\sum_{j=1}^{q} \int_{0}^{T} \max \left\{\tilde{h}_{j}(\boldsymbol{x}(t \mid \boldsymbol{\zeta}), \boldsymbol{\zeta}), 0\right\}^{2} d t .
$$

Clearly, $\Delta(\boldsymbol{\zeta})=0$ if and only if $\boldsymbol{\zeta} \in \Lambda$.

Let $\bar{\epsilon}>0$ be a given constant. We consider the following penalty function defined on $\Gamma \times[0, \bar{\epsilon}]$ :

$$
G_{\sigma}(\boldsymbol{\zeta}, \epsilon)= \begin{cases}J(\boldsymbol{\zeta}), & \text { if } \epsilon=0, \Delta(\boldsymbol{\zeta})=0 \\ J(\boldsymbol{\zeta})+\epsilon^{-\alpha} \Delta(\boldsymbol{\zeta})+\sigma \epsilon^{\beta}, & \text { if } \epsilon \in(0, \bar{\epsilon}], \\ \infty, & \text { if } \epsilon=0, \Delta(\boldsymbol{\zeta}) \neq 0\end{cases}
$$

where $\epsilon \in[0, \bar{\epsilon}]$ is a new decision variable, $\alpha$ and $\beta$ are fixed constants such that $1 \leq \beta \leq \alpha$, and $\sigma>0$ is a penalty parameter.

The penalty function $G_{\sigma}$ is designed to penalize large values of $\Delta(\boldsymbol{\zeta})$. Hence, minimizing $G_{\sigma}$ will likely lead to feasible points satisfying constraints (8) and (9). On this basis, Problem $\mathrm{P}$ can be approximated by the following penalty problem.

Problem Q. Choose $(\boldsymbol{\zeta}, \epsilon) \in \Gamma \times[0, \bar{\epsilon}]$ to minimize the penalty function $G_{\sigma}(\zeta, \epsilon)$.

In the next section, we will present two important convergence results that link Problem Q with Problem P.

Although the definition of the penalty function $G_{\sigma}$ involves three different cases, only the case $\epsilon \in(0, \bar{\epsilon}]$ is of interest in practical computation. The idea is to minimize $G_{\sigma}=J(\boldsymbol{\zeta})+\epsilon^{-\alpha} \Delta(\boldsymbol{\zeta})+\sigma \epsilon^{\beta}$ for increasing values of $\sigma$, stopping once the optimal value of $\epsilon$ is sufficiently small. Thus, in practice, we solve the following optimization problem for an increasing sequence of penalty parameters.

Problem R. Choose $(\boldsymbol{\zeta}, \epsilon) \in \Gamma \times(0, \bar{\epsilon}]$ to minimize the penalty function

$$
G_{\sigma}(\boldsymbol{\zeta}, \epsilon)=J(\boldsymbol{\zeta})+\epsilon^{-\alpha} \Delta(\boldsymbol{\zeta})+\sigma \epsilon^{\beta} .
$$

In Problem R, $G_{\sigma}$ is defined as the sum of three terms - the first term penalizes system cost, the second term penalizes constraint violations, and the third term penalizes large values of $\epsilon$. When $\sigma$ is large, minimizing $G_{\sigma}$ forces $\epsilon$ to be small, which in turn causes $\epsilon^{-\alpha}$ to become large, and thus constraint violations are penalized very severely. Therefore, when $\sigma$ is large, Problem $\mathrm{R}$ is a good approximation of Problem P.

Problem R only involves bound constraints and is therefore much easier to solve than Problem P. Problem R can actually be viewed as a nonlinear programming problem in which the feedback control parameters $\zeta_{k}, k=1, \ldots, m$ and the new decision variable $\epsilon$ need to be chosen to minimize the penalty 
function $G_{\sigma}$. Numerical algorithms for solving such problems typically use the gradient of the cost function to compute descent directions that lead to profitable areas of the search space $[2,18,19]$. Notice, however, that $\boldsymbol{\zeta}$ influences $G_{\sigma}$ implicitly through the dynamic system (7), and thus computing the gradient of $G_{\sigma}$ is not straightforward. In the following, we develop a gradient computation method based on the techniques described by Vincent and Grantham [23], Loxton et al. [14,15], and Kaya and Noakes [9].

First, for each $k=1, \ldots, m$, consider the following variational system:

$$
\begin{aligned}
\dot{\phi}^{k}(t) & =\frac{\partial \tilde{\boldsymbol{f}}(\boldsymbol{x}(t \mid \boldsymbol{\zeta}), \boldsymbol{\zeta})}{\partial \boldsymbol{x}} \boldsymbol{\phi}^{k}(t)+\frac{\partial \tilde{\boldsymbol{f}}(\boldsymbol{x}(t \mid \boldsymbol{\zeta}), \boldsymbol{\zeta})}{\partial \zeta_{k}} \\
\phi^{k}(0) & =\mathbf{0}
\end{aligned}
$$

where

$$
\begin{aligned}
\frac{\partial \tilde{\boldsymbol{f}}(\boldsymbol{x}(t \mid \boldsymbol{\zeta}), \boldsymbol{\zeta})}{\partial \boldsymbol{x}}= & \frac{\partial \boldsymbol{f}(\boldsymbol{x}(t \mid \boldsymbol{\zeta}), \boldsymbol{\varphi}(\boldsymbol{x}(t \mid \boldsymbol{\zeta}), \boldsymbol{\zeta}))}{\partial \boldsymbol{x}} \\
& +\frac{\partial \boldsymbol{f}(\boldsymbol{x}(t \mid \boldsymbol{\zeta}), \boldsymbol{\varphi}(\boldsymbol{x}(t \mid \boldsymbol{\zeta}), \boldsymbol{\zeta}))}{\partial \boldsymbol{u}} \frac{\partial \varphi(\boldsymbol{x}(t \mid \boldsymbol{\zeta}), \boldsymbol{\zeta})}{\partial \boldsymbol{x}}
\end{aligned}
$$

and

$$
\frac{\partial \tilde{\boldsymbol{f}}(\boldsymbol{x}(t \mid \boldsymbol{\zeta}), \boldsymbol{\zeta})}{\partial \zeta_{k}}=\frac{\partial \boldsymbol{f}(\boldsymbol{x}(t \mid \boldsymbol{\zeta}), \boldsymbol{\varphi}(\boldsymbol{x}(t \mid \boldsymbol{\zeta}), \boldsymbol{\zeta}))}{\partial \boldsymbol{u}} \frac{\partial \boldsymbol{\varphi}(\boldsymbol{x}(t \mid \boldsymbol{\zeta}), \boldsymbol{\zeta})}{\partial \zeta_{k}}
$$

Let $\boldsymbol{\phi}^{k}(\cdot \mid \boldsymbol{\zeta})$ denote the solution of (11)-(12). We have the following result.

Theorem 1 For each $\zeta \in \Gamma$,

$$
\frac{\partial \boldsymbol{x}(t \mid \boldsymbol{\zeta})}{\partial \zeta_{k}}=\boldsymbol{\phi}^{k}(t \mid \boldsymbol{\zeta}), \quad t \in[0, T], \quad k=1, \ldots, m .
$$

Proof Since $\boldsymbol{\zeta}$ is fixed throughout this proof, we write $\boldsymbol{x}(\cdot)$ instead of $\boldsymbol{x}(\cdot \mid \boldsymbol{\zeta})$ for simplicity.

First, note that

$$
\frac{\partial}{\partial \zeta_{k}}\{\boldsymbol{x}(0)\}=\frac{\partial}{\partial \zeta_{k}}\left\{\boldsymbol{x}^{0}\right\}=\mathbf{0} .
$$

Thus, $\partial \boldsymbol{x}(\cdot \mid \boldsymbol{\zeta}) / \partial \zeta_{k}$ satisfies the initial condition (12).

Now, by (7),

$$
\boldsymbol{x}(t)=\boldsymbol{x}(0)+\int_{0}^{t} \tilde{\boldsymbol{f}}(\boldsymbol{x}(s), \boldsymbol{\zeta}) d s=\boldsymbol{x}^{0}+\int_{0}^{t} \tilde{\boldsymbol{f}}(\boldsymbol{x}(s), \boldsymbol{\zeta}) d s .
$$

It can be shown that for each fixed $t \in[0, T], \boldsymbol{x}(t \mid \boldsymbol{\zeta})$ is a continuously differentiable function of $\zeta_{k}, k=1, \ldots, m$ (see [15]). Hence, by using Leibniz's rule to differentiate equation (16) with respect to $\zeta_{k}$, we obtain

$$
\frac{\partial \boldsymbol{x}(t)}{\partial \zeta_{k}}=\int_{0}^{t}\left\{\frac{\partial \tilde{\boldsymbol{f}}(\boldsymbol{x}(s), \boldsymbol{\zeta})}{\partial \boldsymbol{x}} \frac{\partial \boldsymbol{x}(s)}{\partial \zeta_{k}}+\frac{\partial \tilde{\boldsymbol{f}}(\boldsymbol{x}(s), \boldsymbol{\zeta})}{\partial \zeta_{k}}\right\} d s,
$$


where $\partial \tilde{\boldsymbol{f}} / \partial \boldsymbol{x}$ and $\partial \tilde{\boldsymbol{f}} / \partial \zeta_{k}$ are as defined in (13) and (14). Differentiating the above equation with respect to time yields

$$
\frac{d}{d t}\left\{\frac{\partial \boldsymbol{x}(t)}{\partial \zeta_{k}}\right\}=\frac{\partial \tilde{\boldsymbol{f}}(\boldsymbol{x}(t), \boldsymbol{\zeta})}{\partial \boldsymbol{x}} \frac{\partial \boldsymbol{x}(t)}{\partial \zeta_{k}}+\frac{\partial \tilde{\boldsymbol{f}}(\boldsymbol{x}(t), \boldsymbol{\zeta})}{\partial \zeta_{k}} .
$$

Equations (15) and (17) show that $\partial \boldsymbol{x}(\cdot \mid \boldsymbol{\zeta}) / \partial \zeta_{k}$ is the solution of the variational system (11)-(12), as required.

On the basis of Theorem 1, the partial derivatives of $G_{\sigma}$ can be determined using standard differentiation rules.

Theorem 2 In Problem $R$, the partial derivatives of $G_{\sigma}$ are given by

$$
\frac{\partial G_{\sigma}(\boldsymbol{\zeta}, \epsilon)}{\partial \zeta_{k}}=\frac{\partial J(\boldsymbol{\zeta})}{\partial \zeta_{k}}+\epsilon^{-\alpha} \frac{\partial \Delta(\boldsymbol{\zeta})}{\partial \zeta_{k}}, \quad k=1, \ldots, m
$$

and

$$
\frac{\partial G_{\sigma}(\boldsymbol{\zeta}, \epsilon)}{\partial \epsilon}=-\alpha \epsilon^{-\alpha-1} \Delta(\boldsymbol{\zeta})+\beta \sigma \epsilon^{\beta-1},
$$

where

$$
\begin{aligned}
\frac{\partial J(\boldsymbol{\zeta})}{\partial \zeta_{k}}= & \frac{\partial \Phi(\boldsymbol{x}(T \mid \boldsymbol{\zeta}), \boldsymbol{\zeta})}{\partial \boldsymbol{x}} \phi^{k}(T \mid \boldsymbol{\zeta})+\frac{\partial \Phi(\boldsymbol{x}(T \mid \boldsymbol{\zeta}), \boldsymbol{\zeta})}{\partial \zeta_{k}} \\
& +\int_{0}^{T}\left\{\frac{\partial \mathcal{L}(\boldsymbol{x}(t \mid \boldsymbol{\zeta}), \boldsymbol{\zeta})}{\partial \boldsymbol{x}} \boldsymbol{\phi}^{k}(t \mid \boldsymbol{\zeta})+\frac{\partial \mathcal{L}(\boldsymbol{x}(t \mid \boldsymbol{\zeta}), \boldsymbol{\zeta})}{\partial \zeta_{k}}\right\} d t \\
\frac{\partial \Delta(\boldsymbol{\zeta})}{\partial \zeta_{k}}=2 \sum_{i=1}^{p} \Psi_{i}(\boldsymbol{x}(T \mid \boldsymbol{\zeta})) \frac{\partial \Psi_{i}(\boldsymbol{x}(T \mid \boldsymbol{\zeta}))}{\partial \boldsymbol{x}} \boldsymbol{\phi}^{k}(T \mid \boldsymbol{\zeta}) & +2 \sum_{j=1}^{q} \int_{0}^{T} \max \left\{\tilde{h}_{j}(\boldsymbol{x}(t \mid \boldsymbol{\zeta}), \boldsymbol{\zeta}), 0\right\} \\
& \times\left\{\frac{\partial \tilde{h}_{j}(\boldsymbol{x}(t \mid \boldsymbol{\zeta}), \boldsymbol{\zeta})}{\partial \boldsymbol{x}} \boldsymbol{\phi}^{k}(t \mid \boldsymbol{\zeta})+\frac{\partial \tilde{h}_{j}(\boldsymbol{x}(t \mid \boldsymbol{\zeta}), \boldsymbol{\zeta})}{\partial \zeta_{k}}\right\} d t .
\end{aligned}
$$

Proof The result can be proved readily by applying standard differentiation rules to equation (10).

On the basis of Theorem 2, we can compute the gradient of $G_{\sigma}$ using the following procedure:

(1) Combine the control system (7) with the variational system (11) to form an expanded system with initial conditions (2) and (12).

(2) Solve the expanded system using any numerical integration scheme.

(3) Compute the partial derivatives of $G_{\sigma}$ using equations (18) and (19).

This procedure can be integrated with a standard nonlinear optimization method - e.g. sequential quadratic programming [18] — to solve Problem R as a nonlinear programming problem. 


\section{Convergence Results}

In this section, we briefly describe the mathematical theory relating Problem $\mathrm{P}$ with Problem Q. We begin with the following result proved in [13].

Theorem 3 Let $\left\{\sigma_{l}\right\}_{l=1}^{\infty}$ be an increasing sequence of penalty parameters such that $\sigma_{l} \rightarrow \infty$ as $l \rightarrow \infty$. Furthermore, let $\left(\boldsymbol{\zeta}^{l, *}, \epsilon^{l, *}\right)$ denote a global solution of Problem Q. Then any limit point of the sequence $\left\{\left(\boldsymbol{\zeta}^{l, *}, \epsilon^{l, *}\right)\right\}_{l=1}^{\infty}$ is a global solution of Problem $P$.

Theorem 3 suggests that we can obtain a solution of Problem P by solving Problem Q sequentially for increasing values of the penalty parameter. As mentioned in the previous section, Problem Q is essentially a nonlinear programming problem that can be solved using standard numerical optimization techniques.

One disadvantage of Theorem 3 is that it requires a global solution of Problem Q. Problem Q is non-convex in general, and thus we will usually only be able to solve it locally. Nevertheless, by making some mild assumptions, one can show that a local solution of Problem Q converges to a local solution of Problem $\mathrm{P}$ as the penalty parameter increases. This is an important convergence property not shared by the well-known constraint transcription method described in $[10,17,24]$.

We assume that for each feasible point $\zeta \in \Lambda$ of Problem $\mathrm{P}$, the following conditions are satisfied:

(A1) The vectors $\partial \Psi_{i}(\boldsymbol{x}(T \mid \boldsymbol{\zeta})) / \partial \boldsymbol{\zeta}, i=1, \ldots, p$ are linearly independent (when $p \neq 0)$.

(A2) There exists a vector $\left[\eta_{1}, \ldots, \eta_{m}\right]^{\top} \in \mathbb{R}^{m}$ and negative real numbers $\vartheta_{1}<0$ and $\vartheta_{2}<0$ such that

$$
\begin{gathered}
\sum_{k=1}^{m} \eta_{k} \frac{\partial \Psi_{i}(\boldsymbol{x}(T \mid \boldsymbol{\zeta}))}{\partial \zeta_{k}}=0, \quad i=1, \ldots, p, \\
\sum_{k=1}^{m} \eta_{k}\left\{\frac{\partial \tilde{h}_{j}(\boldsymbol{x}(t \mid \boldsymbol{\zeta}), \boldsymbol{\zeta})}{\partial \boldsymbol{x}} \boldsymbol{\phi}^{k}(t \mid \boldsymbol{\zeta})\right. \\
\left.+\frac{\partial \tilde{h}_{j}(\boldsymbol{x}(t \mid \boldsymbol{\zeta}), \boldsymbol{\zeta})}{\partial \zeta_{k}}\right\}<\vartheta_{1}, t \in \mathcal{T}_{j}, j=1, \ldots, q, \\
\eta_{k} \begin{cases}>0, & \text { if } \zeta_{k}=a_{k}, \\
<0, & \text { if } \zeta_{k}=b_{k},\end{cases}
\end{gathered}
$$

where $\mathcal{T}_{j}=\left\{s \in[0, T]: \tilde{h}_{j}(\boldsymbol{x}(s \mid \boldsymbol{\zeta}), \boldsymbol{\zeta}) \geq \vartheta_{2}\right\}$.

(A3) There exists a constant $L>0$ and a neighbourhood $\mathcal{N}$ of $\boldsymbol{\zeta}$ such that for each $\left(\boldsymbol{\zeta}^{\prime}, t\right) \in \mathcal{N} \times[0, T]$,

$$
\max \left\{\tilde{h}_{j}\left(\boldsymbol{x}\left(t \mid \boldsymbol{\zeta}^{\prime}\right), \boldsymbol{\zeta}^{\prime}\right), 0\right\}^{2} \leq L \int_{0}^{T} \max \left\{\tilde{h}_{j}\left(\boldsymbol{x}\left(s \mid \boldsymbol{\zeta}^{\prime}\right), \boldsymbol{\zeta}^{\prime}\right), 0\right\}^{2} d s, j=1, \ldots, q .
$$


Under Assumptions (A1)-(A3), we have the following result proved in [13].

Theorem 4 Let $\left\{\sigma_{l}\right\}_{l=1}^{\infty}$ and $\left(\boldsymbol{\zeta}^{l, *}, \epsilon^{l, *}\right)$ be as defined in Theorem 3. Suppose that $\left\{G_{\sigma_{l}}\left(\boldsymbol{\zeta}^{l, *}, \epsilon^{l, *}\right)\right\}_{l=1}^{\infty}$ is bounded. Then there exists a positive integer $l^{\prime}$ such that for each $l \geq l^{\prime}, \boldsymbol{\zeta}^{l, *}$ is a local solution of Problem P.

Theorem 4 implies that when the penalty parameter $\sigma$ is sufficiently large, the values of $\zeta_{k}, k=1, \ldots, m$ in a locally optimal solution of Problem Q will also be locally optimal for Problem P. On this basis, we propose the following algorithm for solving Problem P:

(1) Choose $\boldsymbol{\zeta}^{0} \in \Gamma$ (initial guess), $\sigma^{0}>0$ (initial penalty parameter), $\rho>0$ (tolerance), and $\sigma_{\max }>\sigma^{0}$ (upper bound for the penalty parameter).

(2) Set $\bar{\epsilon} \rightarrow \epsilon^{0}$ and $\sigma^{0} \rightarrow \sigma$.

(3) Starting with $\left(\boldsymbol{\zeta}^{0}, \epsilon^{0}\right)$ as the initial guess, use a nonlinear programming algorithm to solve Problem R. Let $\left(\boldsymbol{\zeta}^{*}, \epsilon^{*}\right)$ denote the local minimizer obtained.

(4) If $\epsilon^{*}<\rho$, then stop: take $\boldsymbol{\zeta}^{*}$ as a solution of Problem P. Otherwise, set $10 \sigma \rightarrow \sigma$ and go to Step 5 .

(5) If $\sigma \leq \sigma_{\max }$, then set $\left(\boldsymbol{\zeta}^{*}, \epsilon^{*}\right) \rightarrow\left(\boldsymbol{\zeta}^{0}, \epsilon^{0}\right)$ and go to Step 3. Otherwise stop: the algorithm cannot find a solution of Problem P.

\section{Example 1: Glider Control (Linear Feedback)}

References [11,12] consider a hang glider whose motion is described by the following system of ordinary differential equations:

$$
\begin{aligned}
& \dot{x}_{1}=x_{3} \cos \left(x_{4}\right), \\
& \dot{x}_{2}=x_{3} \sin \left(x_{4}\right), \\
& \dot{x}_{3}=-\left(k_{1}+k_{2} u^{2}\right) x_{3}^{2}-g \sin \left(x_{4}\right), \\
& \dot{x}_{4}=k_{3} x_{3} u-\frac{g}{x_{3}} \cos \left(x_{4}\right),
\end{aligned}
$$

and

$$
x_{1}(0)=0, x_{2}(0)=0, x_{3}(0)=370, x_{4}(0)=1.5,
$$

where $x_{1}$ is the glider's horizontal position $(\mathrm{m}), x_{2}$ is the glider's altitude $(\mathrm{m})$, $x_{3}$ is the glider's speed $\left(\mathrm{ms}^{-1}\right), x_{4}$ is the angle between the glider's velocity vector and the horizon (radians), $u$ is the glider's angle of attack (radians), $g=9.8$ is the gravitational acceleration $\left(\mathrm{ms}^{-2}\right)$, and $k_{1}, k_{2}$, and $k_{3}$ are model constants defined by

$$
k_{1}=3.289 \times 10^{-5}, k_{2}=1.133 \times 10^{-3}, k_{3}=3.289 \times 10^{-3} .
$$

The aim here is to vary the angle of attack during flight so that the glider's range is maximized. The glider will stall if its angle of attack exceeds operational limits. Hence, we impose the following bound constraints on the angle of attack:

$$
-0.2 \leq u(t) \leq 0.2, \quad t \geq 0 .
$$


The numerical results in [12] show that the glider slows rapidly after launchin the first 50 seconds of flight, the glider's speed drops from a supersonic launch speed of $370 \mathrm{~ms}^{-1}$ to a subsonic speed of $120 \mathrm{~ms}^{-1}$. The speed then oscillates around $120 \mathrm{~ms}^{-1}$ for the remainder of the flight. Hence, the glider's motion consists of two modes: an initial transition mode in which the speed changes from supersonic to subsonic, followed by a steady subsonic mode.

Consider the following linear feedback controller:

$$
u(t)=\zeta_{1} x_{3}(t) \chi_{[0, \tau)}(t)+\zeta_{2} x_{3}(t) \chi_{[\tau, T]}(t),
$$

where $\tau$ is a switching time, $\zeta_{1}$ and $\zeta_{2}$ are feedback gains, and, for a given interval $\mathcal{I} \subset \mathbb{R}$, the indicator function $\chi_{\mathcal{I}}$ is defined by

$$
\chi_{\mathcal{I}}(t)= \begin{cases}1, & \text { if } t \in \mathcal{I} \\ 0, & \text { otherwise }\end{cases}
$$

Note that the feedback gain in (23) changes at $t=\tau$. Thus, this control structure allows different feedback gains for the transition and subsonic modes.

Substituting (23) into (20) gives the following closed-loop system:

$$
\begin{aligned}
& \dot{x}_{1}=x_{3} \cos \left(x_{4}\right), \\
& \dot{x}_{2}=x_{3} \sin \left(x_{4}\right), \\
& \dot{x}_{3}=-\left(k_{1}+k_{2} \zeta_{l}^{2} x_{3}^{2}\right) x_{3}^{2}-g \sin \left(x_{4}\right), \\
& \dot{x}_{4}=k_{3} \zeta_{l} x_{3}^{2}-\frac{g}{x_{3}} \cos \left(x_{4}\right),
\end{aligned}
$$

where $l=1$ for $t<\tau$, and $l=2$ for $t \geq \tau$. Note that (25) is a type of switched system in which the system mode changes at $t=\tau[8,16,25]$.

The bound constraints (22) become

$$
-0.2 \leq \zeta_{1} x_{3}(t) \leq 0.2, \quad t \in[0, \tau),
$$

and

$$
-0.2 \leq \zeta_{2} x_{3}(t) \leq 0.2, \quad t \in[\tau, T] .
$$

Let the terminal time $T$ be the time at which the glider hits the ground (here, $T$ is a free decision variable). Then we have the following terminal state constraint:

$$
x_{2}(T)=0 .
$$

To ensure that $T$ is the first time at which (28) is satisfied, we impose the following continuous inequality constraint:

$$
x_{2}(t)>0, \quad t \in(0, T),
$$

Our optimal feedback control problem is stated as follows: Choose the switching time $\tau$, the feedback gains $\zeta_{1}$ and $\zeta_{2}$, and the terminal time $T$ to maximize the glider's range $x_{1}(T)$ subject to the closed-loop system (25), the initial conditions (21), and the state constraints (26)-(29). 
In this problem, the switching time $\tau$ is a decision variable to be chosen optimally. It is well-known in the optimal control literature that variable switching times cause major problems in numerical computation $[4,14,16,25]$. Thus, we apply the so-called time-scaling transformation, which is described in $[14,16,25]$, to map $\tau$ and $T$ to fixed points in a new time horizon.

Let $s \in[0,2]$ be a new time variable such that

$$
\frac{d t(s)}{d s}=\theta_{1} \chi_{[0,1)}(s)+\theta_{2} \chi_{[1,2]}(s), \quad t(0)=0,
$$

where $\chi_{[0,1)}$ and $\chi_{[1,2]}$ are as defined in (24), and $\theta_{1}$ and $\theta_{2}$ are the durations of the first and second control modes, respectively. Clearly,

$$
t(s)= \begin{cases}\theta_{1} s, & \text { if } s \in[0,1), \\ \theta_{1}+\theta_{2}(s-1), & \text { if } s \in[1,2],\end{cases}
$$

and

$$
t(1)=\theta_{1}, \quad t(2)=\theta_{1}+\theta_{2} .
$$

Applying (30) to the closed-loop system (25) yields

$$
\begin{aligned}
& \dot{x}_{1}=\theta_{l} x_{3} \cos \left(x_{4}\right), \\
& \dot{x}_{2}=\theta_{l} x_{3} \sin \left(x_{4}\right), \\
& \dot{x}_{3}=-\theta_{l}\left(k_{1}+k_{2} \zeta_{l}^{2} x_{3}^{2}\right) x_{3}^{2}-g \theta_{l} \sin \left(x_{4}\right), \\
& \dot{x}_{4}=k_{3} \theta_{l} \zeta_{l} x_{3}^{2}-\frac{g \theta_{l}}{x_{3}} \cos \left(x_{4}\right),
\end{aligned}
$$

where $l=1$ for $s \in[0,1), l=2$ for $s \in[1,2]$, and the overhead dot denotes differentiation with respect to $s$. Furthermore, the state constraints (26)-(29) become

$$
-0.2 \leq \zeta_{1} x_{3}(s) \chi_{[0,1)}(s)+\zeta_{2} x_{3}(s) \chi_{[1,2]}(s) \leq 0.2
$$

and

$$
x_{2}(2)=0, \quad x_{2}(s)>0, \quad s \in(0,2) .
$$

The switching time and terminal time in (31) are fixed. Hence, this transformed system is much easier to work with than the original system.

We approximate the open constraint (33) by

$$
x_{2}(2)=0, \quad x_{2}(s) \geq \delta, \quad s \in[\varsigma, 2-\varsigma],
$$

where $\delta>0$ and $\varsigma>0$ are small positive constants. We now define an approximate optimal control problem as follows: Choose $\theta_{1}, \theta_{2}, \zeta_{1}$, and $\zeta_{2}$ to maximize the glider's range $x_{1}(2)$ subject to the closed-loop system (31), the initial conditions (21), and the constraints (32) and (34).

To solve this problem, we wrote a Fortran program based on the exact penalty approach described in the previous sections. This program uses NLPQLP [22] to solve the penalty problem, and the Runge-Kutta order 6 


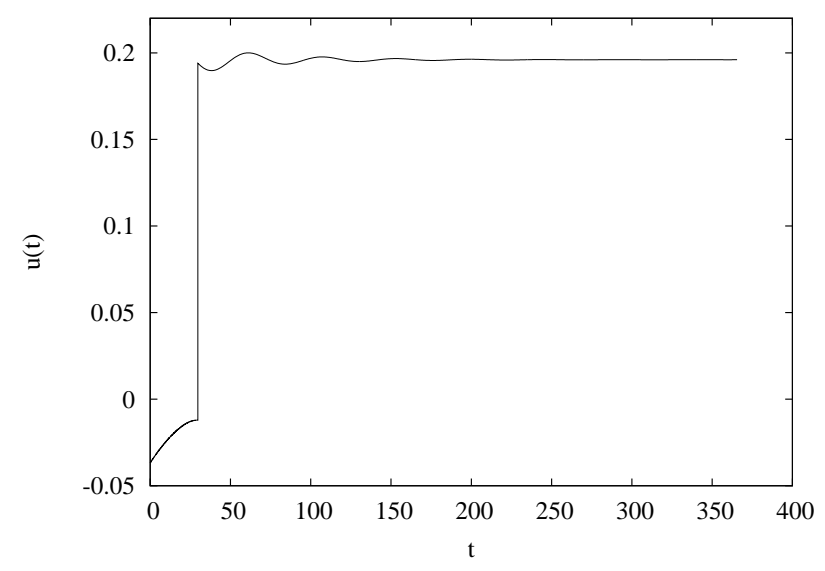

Fig. 1 Optimal control for Example 1.

method to solve the differential equations. The optimal solution generated by our program is

$$
\begin{aligned}
& \theta_{1}^{*}=29.3780, \quad \theta_{2}^{*}=336.1364, \\
& \zeta_{1}^{*}=-9.9429 \times 10^{-5}, \quad \zeta_{2}^{*}=1.5957 \times 10^{-3} .
\end{aligned}
$$

This solution corresponds to an optimal switching time of $\tau^{*}=\theta_{1}^{*}=29.3780$ and an optimal terminal time of $T^{*}=\theta_{1}^{*}+\theta_{2}^{*}=365.5144$. The glider's maximum range is 44,120 metres. The optimal feedback control is shown in Figure 1 , and the corresponding flight trajectory and airspeed plots are shown in Figures 2 and 3.

\section{Example 2: Glider Control (Nonlinear Feedback)}

Consider system (20)-(21) with the following nonlinear feedback controller:

$$
u(t)=\zeta_{1} x_{3}(t) \cos \left(x_{4}(t)\right) \chi_{[0, \tau)}(t)+\zeta_{2} x_{3}(t) \cos \left(x_{4}(t)\right) \chi_{[\tau, T]}(t),
$$

where $\tau$ is a switching time, $\zeta_{1}$ and $\zeta_{2}$ are feedback gains, and $\chi_{[0, \tau)}$ and $\chi_{[\tau, T]}$ are as defined in (24). The controller (35) expresses the angle of attack as a function of the vertical speed, rather than the total speed. Such control laws are typically used for gliders.

Substituting (35) into (20) gives

$$
\begin{aligned}
& \dot{x}_{1}=x_{3} \cos \left(x_{4}\right), \\
& \dot{x}_{2}=x_{3} \sin \left(x_{4}\right), \\
& \dot{x}_{3}=-\left(k_{1}+k_{2} \zeta_{l}^{2} x_{3}^{2} \cos ^{2}\left(x_{4}\right)\right) x_{3}^{2}-g \sin \left(x_{4}\right), \\
& \dot{x}_{4}=k_{3} \zeta_{l} x_{3}^{2} \cos \left(x_{4}\right)-\frac{g}{x_{3}} \cos \left(x_{4}\right),
\end{aligned}
$$




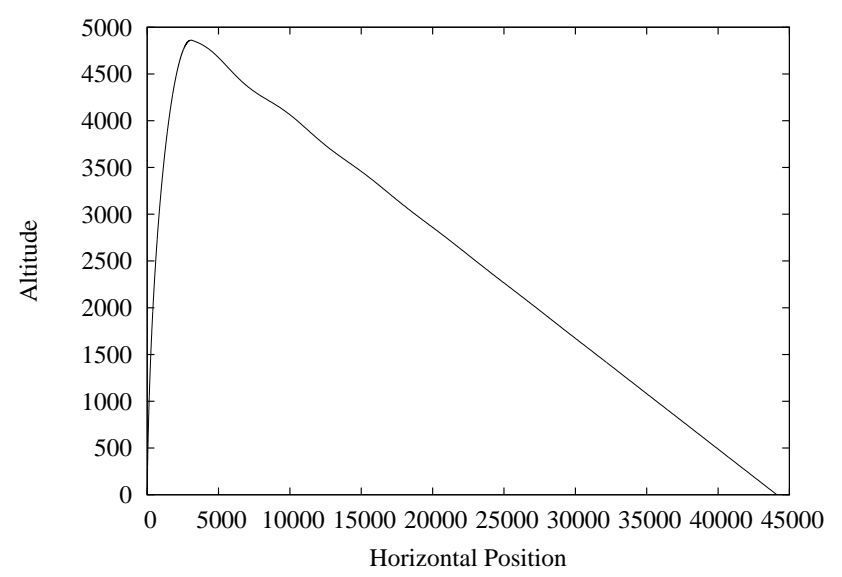

Fig. 2 Optimal flight trajectory for Example 1.

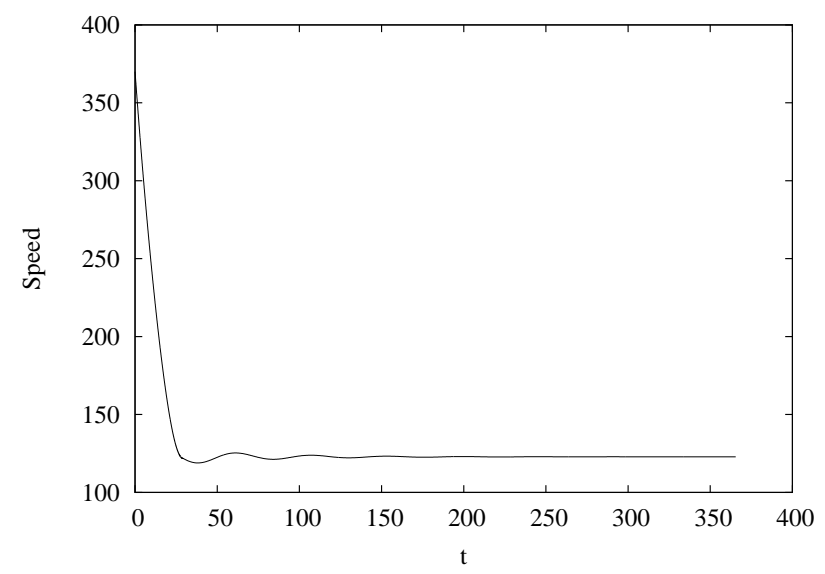

Fig. 3 Airspeed plot for Example 1.

where $l=1$ for $t<\tau$ and $l=2$ for $t \geq \tau$. The bound constraints for the angle of attack become

$$
-0.2 \leq \zeta_{1} x_{3}(t) \cos \left(x_{4}(t)\right) \leq 0.2, \quad t \in[0, \tau),
$$

and

$$
-0.2 \leq \zeta_{2} x_{3}(t) \cos \left(x_{4}(t)\right) \leq 0.2, \quad t \in[\tau, T] .
$$

Our optimal feedback control problem is: Choose the switching time $\tau$, the feedback gains $\zeta_{1}$ and $\zeta_{2}$, and the terminal time $T$ to maximize the glider's range $x_{1}(T)$ subject to the closed-loop system (36), the initial conditions (21), and the state constraints (28)-(29) and (37)-(38). 
As in Example 1, we introduce a new time variable $s \in[0,2]$ and apply the time-scaling transformation to yield the following transformed system:

$$
\begin{aligned}
& \dot{x}_{1}=\theta_{l} x_{3} \cos \left(x_{4}\right), \\
& \dot{x}_{2}=\theta_{l} x_{3} \sin \left(x_{4}\right), \\
& \dot{x}_{3}=-\theta_{l}\left(k_{1}+k_{2} \zeta_{l}^{2} x_{3}^{2} \cos ^{2}\left(x_{4}\right)\right) x_{3}^{2}-g \theta_{l} \sin \left(x_{4}\right), \\
& \dot{x}_{4}=k_{3} \theta_{l} \zeta_{l} x_{3}^{2} \cos \left(x_{4}\right)-\frac{g \theta_{l}}{x_{3}} \cos \left(x_{4}\right),
\end{aligned}
$$

where, as in Example 1, $\theta_{1}$ is the duration of control mode 1 and $\theta_{2}$ is the duration of control mode 2 . Under the time-scaling transformation, the constraints (37)-(38) become

$$
-0.2 \leq \zeta_{1} x_{3}(s) \cos \left(x_{4}(s)\right) \leq 0.2, \quad s \in[0,1),
$$

and

$$
-0.2 \leq \zeta_{2} x_{3}(s) \cos \left(x_{4}(s)\right) \leq 0.2, \quad s \in[1,2] .
$$

Thus, our approximate problem is: Choose $\theta_{1}, \theta_{2}, \zeta_{1}$, and $\zeta_{2}$ to maximize the glider's range $x_{1}(2)$ subject to the closed-loop system (39), the initial conditions (21), and the constraints (34) and (40)-(41).

We solved this problem using NLPQLP in conjunction with our new exact penalty approach. The optimal solution is

$$
\begin{aligned}
& \theta_{1}^{*}=28.2150, \quad \theta_{2}^{*}=333.8295, \\
& \zeta_{1}^{*}=-1.2598 \times 10^{-4}, \quad \zeta_{2}^{*}=-1.3928 \times 10^{-2} .
\end{aligned}
$$

The corresponding optimal switching time and optimal terminal time are

$$
\tau^{*}=\theta_{1}^{*}=28.2150, \quad T^{*}=\theta_{1}^{*}+\theta_{2}^{*}=362.0445 .
$$

The maximum range is 44,252 metres. The optimal control, flight trajectory, and airspeed plot are shown in Figures 4-6.

\section{Conclusion}

We have presented a new approach for designing optimal feedback control laws for constrained systems. This approach is based on a new exact penalty function, which was first introduced in [27] to solve semi-infinite programming problems. We successfully applied our approach to two examples involving the optimal control of a gliding projectile. As shown through these examples, the exact penalty approach is effective at solving feedback control problems with highly nonlinear dynamics. 


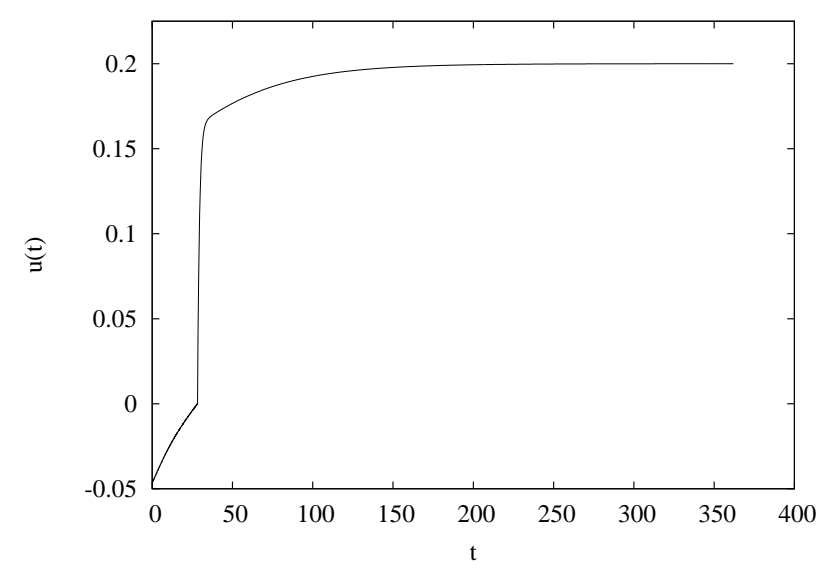

Fig. 4 Optimal control for Example 2.

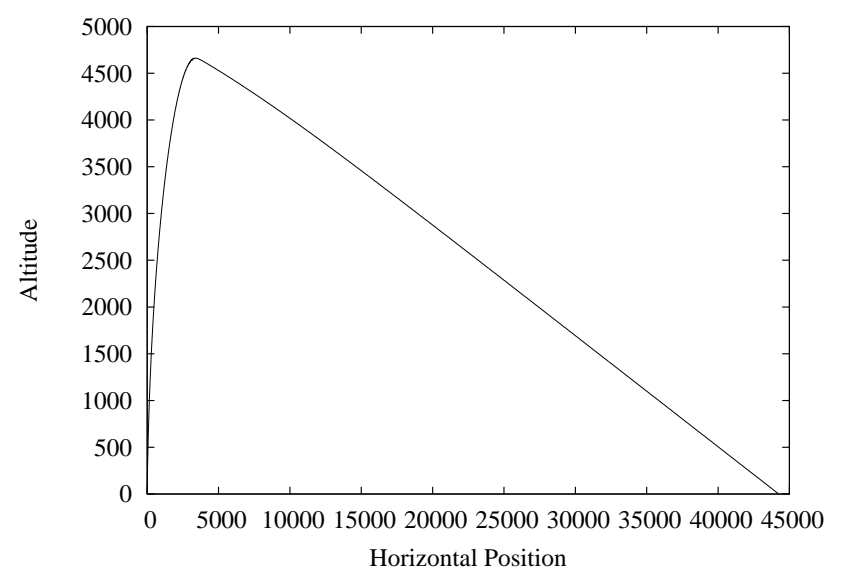

Fig. 5 Optimal flight trajectory for Example 2.

\section{References}

1. B. Açikmeşe and L. Blackmore, Lossless convexification of a class of optimal control problems with non-convex control constraints, Automatica, 47, 341-347 (2011)

2. M. S. Bazarra, H. D. Sherali, and C. M. Shetty, Nonlinear Programming: Theory and Algorithms, New Jersey: John Wiley (2006)

3. R. L. Burden and J. D. Faires, Numerical Analysis, Boston: Brooks Cole, (2010)

4. B. Farhadinia, K. L. Teo, and R. Loxton, A computational method for a class of nonstandard time optimal control problems involving multiple time horizons, Mathematical and Computer Modelling, 49, 1682-1691 (2009)

5. M. E. Fisher, W. J. Grantham, and K. L. Teo, Neighbouring extremals for nonlinear systems with control constraints, Dynamics and Control, 5, 225-240 (1995)

6. M. Gerdts and M. Kunkel, A nonsmooth Newton's method for discretized optimal control problems with state and control constraints, Journal of Industrial and Management 


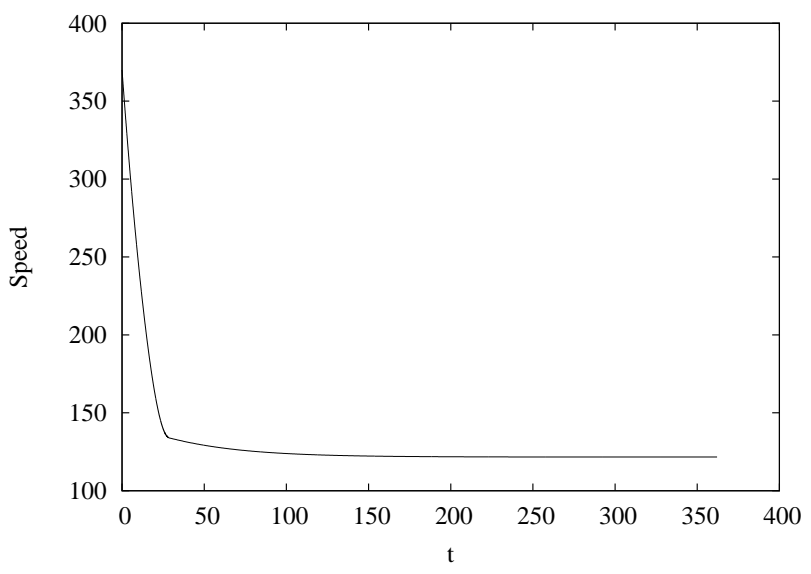

Fig. 6 Airspeed plot for Example 2.

Optimization, 4, 247-270 (2008)

7. C. Jiang, K. L. Teo, R. Loxton, and G. R. Duan, A neighboring extremal solution for an optimal switched impulsive control problem, Journal of Industrial and Management Optimization, 8, 591-609 (2012).

8. M. Kamgarpour and C. Tomlin, On optimal control of non-autonomous switched systems with a fixed mode sequence, Automatica, 48, 1177-1181 (2012)

9. C. Y. Kaya and J. L. Noakes, Computational method for time-optimal switching control, Journal of Optimization Theory and Applications, 117, 69-92 (2003)

10. B. Li, K. L. Teo, C. C. Lim, and G. R. Duan, An optimal PID controller design for nonlinear constrained optimal control problems, Discrete and Continuous Dynamical Systems - Series B, 16, 1101-1117 (2011).

11. Q. Lin, R. Loxton, K. L. Teo, and Y. H. Wu, A new computational method for a class of free terminal time optimal control problems, Pacific Journal of Optimization, 7, 63-81 (2011)

12. Q. Lin, R. Loxton, K. L. Teo, and Y. H. Wu, Optimal control computation for nonlinear systems with state-dependent stopping criteria, Automatica, 48, 2116-2129 (2012)

13. Q. Lin, R. Loxton, K. L. Teo, Y. H. Wu, and C. Yu, A new exact penalty method for semi-infinite programming problems, Journal of Computational and Applied Mathematics, accepted subject to minor changes.

14. R. Loxton, K. L. Teo, and V. Rehbock, Optimal control problems with multiple characteristic time points in the objective and constraints, Automatica, 44, 2923-2929 (2008)

15. R. Loxton, K. L. Teo, and V. Rehbock, Robust suboptimal control of nonlinear systems, Applied Mathematics and Computation, 217, 6566-6576 (2011)

16. R. Loxton, K. L. Teo, V. Rehbock, and W. K. Ling, Optimal switching instants for a switched-capacitor DC/DC power converter, Automatica, 45, 973-980 (2009)

17. R. Loxton, K. L. Teo, V. Rehbock, and K. F. C. Yiu, Optimal control problems with a continuous inequality constraint on the state and the control, Automatica, 45, 2250-2257 (2009)

18. D. G. Luenberger and Y. Ye, Linear and Nonlinear Programming, New York: Springer (2008)

19. J. Nocedal and S. J. Wright, Numerical Optimization, New York: Springer (2006)

20. L. S. Pontryagin, The Mathematical Theory of Optimal Processes, New York: Gordan and Breach (1986)

21. V. Rehbock, K. L. Teo, and L. S. Jennings, A computational procedure for suboptimal robust controls, Dynamics and Control, 2, 331-348 (1992) 
22. K. Schittkowski, NLPQLP: A Fortran Implementation of a Sequential Quadratic Programming Algorithm with Distributed and Non-Monotone Line Search - User's Guide, University of Bayreuth, Germany, (2007)

23. T. L. Vincent and W. J. Grantham, Optimality in Parametric Systems, New York: John Wiley (1981)

24. L. Y. Wang, W. H. Gui, K. L. Teo, R. Loxton, and C. H. Yang, Time delayed optimal control problems with multiple characteristic time points: Computation and industrial applications, Journal of Industrial and Management Optimization, 5, 705-718 (2009)

25. X. Xu and P. J. Antsaklis, Optimal control of switched systems based on parameterization of the switching instants, IEEE Transactions on Automatic Control, 49, 2-16 (2004)

26. C. Yu, B. Li, R. Loxton, and K. L. Teo, Optimal discrete-valued control computation, Journal of Global Optimization, in press.

27. C. Yu, K. L. Teo, L. Zhang, and Y. Bai, A new exact penalty function method for continuous inequality constrained optimization problems, Journal of Industrial and Management Optimization, 6, 895-910 (2010)

28. Y. Zhao and M. A. Stadtherr, Rigorous global optimization method for dynamic systems subject to inequality path constraints, Industrial and Engineering Chemistry Research, 50, 12678-12693 (2011)

29. J. Zhou, K. L. Teo, D. Zhou, and G. Zhao, Nonlinear optimal feedback control for lunar module soft landing, Journal of Global Optimization, 52, 211-227 (2012) 